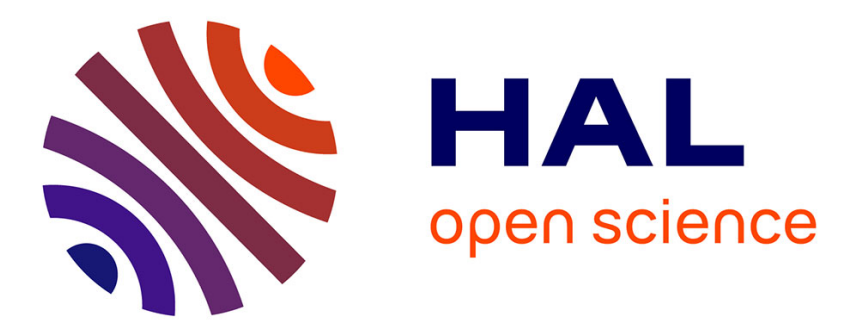

\title{
Stationary solutions of a small gyrostat in the Newtonian field of two bodies with equal masses
}

Tilemahos J. Kalvouridis

\section{To cite this version:}

Tilemahos J. Kalvouridis. Stationary solutions of a small gyrostat in the Newtonian field of two bodies with equal masses. Nonlinear Dynamics, 2010, 61 (3), pp.373-381. 10.1007/s11071-010-9655-0 . hal00560020

\section{HAL Id: hal-00560020 \\ https://hal.science/hal-00560020}

Submitted on 27 Jan 2011

HAL is a multi-disciplinary open access archive for the deposit and dissemination of scientific research documents, whether they are published or not. The documents may come from teaching and research institutions in France or abroad, or from public or private research centers.
L'archive ouverte pluridisciplinaire HAL, est destinée au dépôt et à la diffusion de documents scientifiques de niveau recherche, publiés ou non, émanant des établissements d'enseignement et de recherche français ou étrangers, des laboratoires publics ou privés. 


\title{
Stationary solutions of a small gyrostat in the Newtonian field of two bodies with equal masses
}

\author{
Tilemahos J. Kalvouridis
}

Received: 12 April 2009 / Accepted: 3 January 2010 / Published online: 27 January 2010

(C) Springer Science+Business Media B.V. 2010

\begin{abstract}
The paper deals with the dynamics of a small gyrostat satellite acted upon by the Newtonian forces of two big bodies of equal masses which rotate around their center of mass. The gyrostat's equations of motion are derived and classes of its stationary solutions, as well as their stability are studied.
\end{abstract}

Keywords Gyrostat · Rigid body dynamics .

Stationary solutions $\cdot$ Stability

\section{Introduction}

Problems with gyrostats have been studied in the past, particularly during the last decades $([1-10,12-14]$, etc.). Recently, Vera and Vigueras $[15,16]$ and Vera [17] have renewed the interest on this issue with important results. These problems are extremely interesting, since most of the artificial satellites and spacecrafts have mobile parts such as rotating antennas, photovoltaic panels, or mechanical articulated arms, etc., and can therefore be presumed as gyrostatic bodies. On the other hand, a special case of the old and famous restricted three-body problem is the Copenhagen configuration where the two dominant masses

T.J. Kalvouridis ( $\square)$

Faculty of Applied Sciences, Department of Mechanics,

National Technical University of Athens, Zografou

Campus 157 73, Athens, Greece

e-mail: tkalvouridis@gmail.com are equal. This case has lately gained new scientific interest after the discovery of many exo-solar planetary systems, the majority of which consists of two members. The recent estimations about the partners in such a system show that, in many cases, their masses can roughly be considered as equal. Here, we consider a gyrostat $S$ with infinitesimal mass moving in the Newtonian field of two big spherical and homogeneous bodies $P_{1}$ and $P_{2}$ of equal masses $m$ that rotate about their center of mass with constant angular velocity $\omega$. The much smaller body $S$ of mass $m_{0}$, does not affect the motion of the primaries and has a gyrostatic structure (Fig. 1). As we know, a gyrostat consists of two parts: a rigid part called the platform or carrier and $n$ other parts, the rotors, which are connected to the platform. Each rotor is spinning-independently about an axis fixed on the platform and its motion does not modify the mass distribution of the gyrostat. The platform may rotate about an inertial reference frame, so that a gyrostat is generally characterized by $n+1$ angular velocities, $n$ of which, are the angular velocities of the spinning rotors relative to the platform. Sometimes, in the relevant literature, a gyrostat is also referred to as a 'dual-spin' body. Hereunder, we shall examine gyrostats with only one rotor. First, we extract the equations of motion of the gyrostat and then we investigate the equilibrium states (translational and rotational) and their linear stability assuming that the small body is inertially axisymmetric. The obtained results are illustrated with some figures. 
(S)
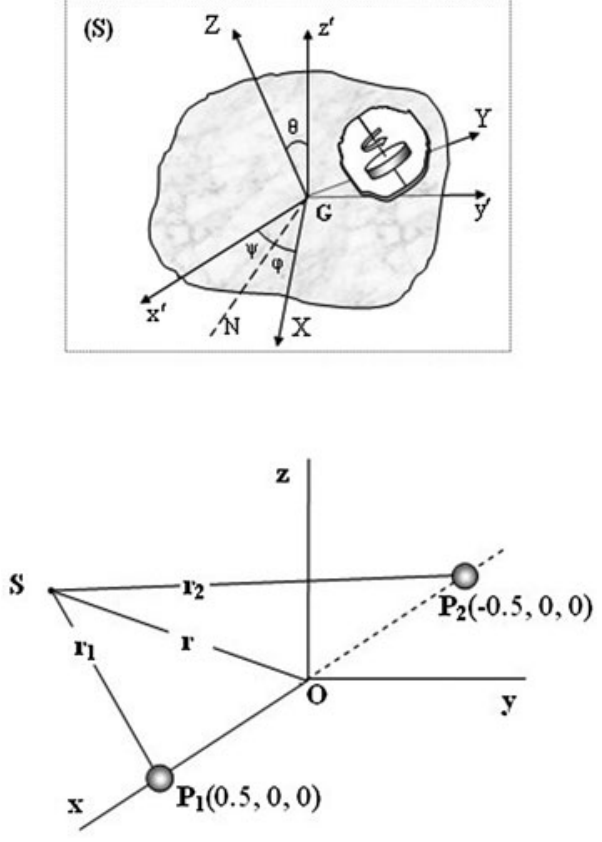

Fig. 1 The configuration of the system. The framed picture shows the gyrostatic structure of the infinitesimal body $S$ and the body-fixed axes. $G N$ is the line of nodes and $O x y z$ is the synodic coordinate system

\section{Equations of motion}

We use a synodic system $O x y z$ (unit vectors $\underline{i}, \underline{j}, \underline{k}$ ) rigidly attached to the system of the two primaries $P_{1}$ and $P_{2}$ and centered at their mass center $O$. Its $x$ axis coincides with the line joining the two primaries, while the $y$-axis is perpendicular to it on the orbital plane of the massive bodies. We assume that the synodic system rotates with a constant angular velocity $\omega$. We also use two auxiliary reference frames centered at the mass center $G$ of $S$; the body-fixed $G X Y Z$ (unit vectors $I, J, K)$ the axes of which coincide with the gyrostat's principal central axes of inertia and $G x^{\prime} y^{\prime} z^{\prime}$, the axes of which are parallel to those of the synodic system (Fig. 1).

In our calculations, we consider the triad of Euler angles $(\psi, \theta, \phi)$ that is defined from the rotating sequence 3-1-3 (Fig. 1). Hence, the components of the angular velocity $\Omega$ of the gyrostat with respect to an inertial frame, can be expressed as follows:

$$
\begin{aligned}
\Omega= & \{(\dot{\psi}+\omega) \sin \phi \sin \theta+\dot{\theta} \cos \phi, \\
& (\dot{\psi}+\omega) \cos \phi \sin \theta-\dot{\theta} \sin \phi, \\
& (\dot{\psi}+\omega) \cos \theta+\dot{\phi}\}
\end{aligned}
$$

The kinetic energy of the translational-rotational motion of the gyrostat is given with the relation:

$$
\begin{aligned}
T= & \frac{1}{2} m_{0}\left[(\dot{x}-\omega y)^{2}+(\dot{y}+\omega x)^{2}+\dot{z}^{2}\right] \\
& +\frac{1}{2}\left(I_{1}-I_{2}\right) \sin \theta \sin 2 \phi \dot{\theta}(\dot{\psi}+\omega) \\
& +\frac{1}{2}\left[\left(I_{1} \sin ^{2} \phi+I_{2} \cos ^{2} \phi\right) \sin ^{2} \theta\right. \\
& \left.+I_{3} \cos ^{2} \theta\right](\dot{\psi}+\omega)^{2}+\frac{1}{2}\left(I_{1} \cos ^{2} \phi\right. \\
& \left.+I_{2} \sin ^{2} \phi\right) \dot{\theta}^{2}+\frac{1}{2} I_{3} \dot{\phi}^{2}+I_{3} \cos \theta \dot{\phi}(\dot{\psi}+\omega) \\
& +\left(h_{a} \cos \phi-h_{b} \sin \phi\right) \dot{\theta}+c \dot{\phi} \\
& +\left[\left(h_{a} \sin \phi+h_{b} \cos \phi\right) \sin \theta\right. \\
& +c \cos \theta](\dot{\psi}+\omega)+T_{r}
\end{aligned}
$$

where $m_{0}$ is the mass of the gyrostat, $I_{i}, i=1,2,3$ are its principal central moments of inertia, $h_{a}, h_{b}, h_{c}$ are the components of the rotor's internal moment of momentum and $T_{r}$ is its kinetic energy relative to the platform. If we assume that the rotor rotates with relation to the carrier with constant angular velocity, then the quantity $T_{r}$ is constant, and consequently it does not appear in the final form of the equations of motion.

The potential energy after MacCullagh's formula [11] can be expressed in terms of Eulerian angles [9]

$$
\begin{aligned}
V= & -G m m_{0}\left[\frac{1}{r_{1}}+\frac{1}{r_{2}}\right] \\
& +G m\left\{\sum _ { i = 1 } ^ { 2 } \frac { 1 } { r _ { i } ^ { 5 } } \left[\left(x-x_{i}\right)^{2} f+y^{2} g+z^{2} u\right.\right. \\
& \left.\left.+\left(x-x_{i}\right) y h+\left(x-x_{i}\right) z w+y z v\right]\right\}
\end{aligned}
$$

where $r_{1}, r_{2}$, are the distances of the gyrostat $S$ from the primaries,

$$
\begin{aligned}
& r_{1}^{2}=\left(x-x_{1}\right)^{2}+y^{2}+z^{2} \\
& r_{2}^{2}=\left(x-x_{2}\right)^{2}+y^{2}+z^{2}
\end{aligned}
$$

and

$$
\begin{aligned}
f= & -\frac{3 p}{2} \sin ^{2} \psi \sin ^{2} \theta+\frac{3 s}{4} \cos ^{2} \psi \cos 2 \phi \\
& -\frac{3 s}{4} \sin ^{2} \psi \cos ^{2} \theta \cos 2 \phi
\end{aligned}
$$




$$
\begin{aligned}
& -\frac{3 s}{4} \sin 2 \psi \cos \theta \sin 2 \phi+\frac{p}{2} \\
g= & -\frac{3 p}{2} \cos ^{2} \psi \sin ^{2} \theta+\frac{3 s}{4} \sin ^{2} \psi \cos 2 \phi \\
& -\frac{3 s}{4} \cos ^{2} \psi \cos ^{2} \theta \cos 2 \phi \\
& +\frac{3 s}{4} \sin 2 \psi \cos \theta \sin 2 \phi+\frac{p}{2} \\
h= & \frac{3 p}{2} \sin 2 \psi \sin ^{2} \theta+\frac{3 s}{4} \sin 2 \psi \cos 2 \phi \\
& +\frac{3 s}{2} \cos 2 \psi \cos \theta \sin 2 \phi \\
& +\frac{3 s}{4} \sin 2 \psi \cos 2 \theta \cos 2 \phi \\
u= & -\frac{3 p}{2} \cos { }^{2} \theta-\frac{3 s}{4} \sin 2 \theta \cos 2 \phi+\frac{p}{2} \\
v= & \frac{3 p}{2} \cos \psi \sin 2 \theta+\frac{3 s}{2} \sin \psi \sin \theta \sin 2 \phi \\
& -\frac{3 s}{4} \cos \psi \sin 2 \theta \cos 2 \phi \\
w= & -\frac{3 p}{2} \sin \psi \sin 2 \theta+\frac{3 s}{2} \cos \psi \sin \theta \sin 2 \phi \\
& +\sin 2 \theta \cos 2 \phi \\
& \\
&
\end{aligned}
$$

with

$p=\frac{I_{1}+I_{2}-2 I_{3}}{2}$ and $s=I_{1}-I_{2}$

The equations of motion are of two kinds: those that express the translational motion of the body and those that describe its rotational motion. Both systems of equations are coupled. More specifically:

I. Translational motion The translational motion is described by means of the equations

$$
\begin{aligned}
& \ddot{x}-2 \omega \dot{y} \\
& =\omega^{2} x-G m\left[\sum_{i=1}^{2} \frac{\left(x-x_{i}\right)}{r_{i}^{3}}\right] \\
& \quad-G \frac{m}{m_{0}}\left\{\sum _ { i = 1 } ^ { 2 } \frac { \partial } { \partial x } \left[\frac { 1 } { r _ { i } ^ { 5 } } \left[\left(x-x_{i}\right)^{2} f+y^{2} g+z^{2} u\right.\right.\right. \\
& \left.\left.\left.+\left(x-x_{i}\right) y h+\left(x-x_{i}\right) z w+y z v\right]\right]\right\}
\end{aligned}
$$

$$
\begin{aligned}
\ddot{y}+ & \omega \dot{x} \\
= & \omega^{2} y-G m\left[\sum_{i=1}^{2} \frac{y}{r_{i}^{3}}\right] \\
& -G \frac{m}{m_{0}}\left\{\sum _ { i = 1 } ^ { 2 } \frac { \partial } { \partial y } \left[\frac { 1 } { r _ { i } ^ { 5 } } \left[\left(x-x_{i}\right)^{2} f+y^{2} g+z^{2} u\right.\right.\right. \\
& \left.\left.\left.+\left(x-x_{i}\right) y h+\left(x-x_{i}\right) z w+y z v\right]\right]\right\} \\
\ddot{z}=- & G m\left[\sum_{i=1}^{2} \frac{z}{r_{i}^{3}}\right] \\
& -G \frac{m}{m_{0}}\left\{\sum _ { i = 1 } ^ { 2 } \frac { \partial } { \partial z } \left[\frac { 1 } { r _ { i } ^ { 5 } } \left[\left(x-x_{i}\right)^{2} f+y^{2} g+z^{2} u\right.\right.\right. \\
& \left.\left.\left.+\left(x-x_{i}\right) y h+\left(x-x_{i}\right) z w+y z v\right]\right]\right\}
\end{aligned}
$$

II. Rotational motion The rotational motion is described by the equations

$$
\begin{aligned}
{\left[\left(I_{1} \sin ^{2} \phi+I_{2} \cos ^{2} \phi\right) \sin ^{2} \theta+I_{3} \cos ^{2} \theta\right] \ddot{\psi} } \\
+\quad \frac{1}{2}\left(I_{1}-I_{2}\right) \sin 2 \phi \sin \theta \ddot{\theta}+I_{3} \cos \theta \ddot{\phi} \\
+\left[\left(I_{1}-I_{2}\right) \sin 2 \phi \sin ^{2} \theta \dot{\phi}+\left(I_{1} \sin ^{2} \phi\right.\right. \\
\left.\left.+I_{2} \cos ^{2} \phi\right) \sin 2 \theta \dot{\theta}-I_{3} \sin 2 \theta \dot{\theta}\right](\dot{\psi}+\omega) \\
+\left(I_{1}-I_{2}\right) \cos 2 \phi \sin \theta \dot{\theta} \dot{\phi} \\
+\frac{1}{2}\left(I_{1}-I_{2}\right) \sin 2 \phi \cos \theta \dot{\theta}^{2}-I_{3} \sin \theta \dot{\theta} \dot{\phi} \\
+\left[\left(h_{a} \sin \phi+h_{b} \cos \phi\right) \cos \theta-h_{c} \sin \theta\right] \dot{\theta} \\
+\left(h_{a} \cos \phi-h_{b} \sin \phi\right) \sin \theta \dot{\phi} \\
=-G m\left\{\sum _ { i = 1 } ^ { 2 } \frac { 1 } { r _ { i } ^ { 5 } } \left[\left(x-x_{i}\right)^{2} \frac{\partial f}{\partial \psi}+y^{2} \frac{\partial g}{\partial \psi}+z^{2} \frac{\partial u}{\partial \psi}\right.\right. \\
\left.\left.\quad+\left(x-x_{i}\right) y \frac{\partial h}{\partial \psi}+\left(x-x_{i}\right) z \frac{\partial w}{\partial \psi}+y z \frac{\partial v}{\partial \psi}\right]\right\} \\
\frac{1}{2}\left(I_{1}-I_{2}\right) \sin 2 \phi \sin \theta \ddot{\psi}+\left(I_{1} \cos ^{2} \phi+I_{2} \sin ^{2} \phi\right) \ddot{\theta} \\
-\left(I_{1}-I_{2}\right) \sin 2 \phi \dot{\theta} \dot{\phi}
\end{aligned}
$$




$$
\begin{aligned}
& +\left(I_{1}-I_{2}\right) \cos 2 \phi \sin \theta \dot{\phi}(\dot{\psi}+\omega) \\
& -\frac{1}{2}\left[\left(I_{1} \sin ^{2} \phi+I_{2} \cos ^{2} \phi\right)-I_{3}\right] \\
& \times \sin 2 \theta(\dot{\psi}+\omega)^{2}+I_{3} \sin \theta \dot{\phi}(\dot{\psi}+\omega) \\
& -\left(h_{a} \sin \phi+h_{b} \cos \phi\right) \dot{\phi} \\
& -\left[\left(h_{a} \sin \phi+h_{b} \cos \phi\right) \cos \theta-h_{c} \sin \theta\right] \\
& \times(\dot{\psi}+\omega) \\
& =-G m\left\{\sum _ { i = 1 } ^ { 2 } \frac { 1 } { r _ { i } ^ { 5 } } \left[\left(x-x_{i}\right)^{2} \frac{\partial f}{\partial \theta}+y^{2} \frac{\partial g}{\partial \theta}+z^{2} \frac{\partial u}{\partial \theta}\right.\right. \\
& \left.\left.+\left(x-x_{i}\right) y \frac{\partial h}{\partial \theta}+\left(x-x_{i}\right) z \frac{\partial w}{\partial \theta}+y z \frac{\partial v}{\partial \theta}\right]\right\}
\end{aligned}
$$

$I_{3} \cos \theta \ddot{\psi}+I_{3} \ddot{\phi}-I_{3} \sin \theta \dot{\theta}(\dot{\psi}+\omega)$

$$
\begin{aligned}
& -\frac{1}{2}\left(I_{1}-I_{2}\right) \sin 2 \phi \sin ^{2} \theta(\dot{\psi}+\omega)^{2} \\
& +\frac{1}{2}\left(I_{1}-I_{2}\right) \sin 2 \phi \dot{\theta}^{2}-\left(I_{1}-I_{2}\right) \cos 2 \phi \\
& \times \sin \theta \dot{\theta}(\dot{\psi}+\omega)-\left(h_{a} \cos \phi-h_{b} \sin \phi\right) \\
& \times \sin \theta(\dot{\psi}+\omega)+\left(h_{a} \sin \phi+h_{b} \cos \phi\right) \dot{\theta} \\
& =-G m\left\{\sum _ { i = 1 } ^ { 2 } \frac { 1 } { r _ { i } ^ { 5 } } \left[\left(x-x_{i}\right)^{2} \frac{\partial f}{\partial \phi}+y^{2} \frac{\partial g}{\partial \phi}\right.\right. \\
& +z^{2} \frac{\partial u}{\partial \phi}+\left(x-x_{i}\right) y \frac{\partial h}{\partial \phi}+\left(x-x_{i}\right) z \frac{\partial w}{\partial \phi} \\
& \left.\left.+y z \frac{\partial v}{\partial \phi}\right]\right\}
\end{aligned}
$$

As we have mentioned before, (1) to (6) are coupled.

\section{Stationary solutions and stability}

Stationary solutions of the gyrostat exist under the conditions $\dot{x}=\dot{y}=\dot{z}=\ddot{x}=\ddot{y}=\ddot{z}=\dot{\theta}=\ddot{\theta}=\dot{\phi}=$ $\ddot{\phi}=\dot{\psi}=\ddot{\psi}=0$. For practical reasons we shall confine our study to those orientations which are determined by all possible combinations of the angles

$$
\begin{aligned}
& \psi=\lambda_{\psi} \frac{\pi}{2} \quad \text { with } \lambda_{\psi}=0,1,2,3 \\
& \theta=\lambda_{\theta} \frac{\pi}{2} \quad \text { with } \lambda_{\theta}=0,1,2 \quad \text { and } \\
& \phi=\lambda_{\phi} \frac{\pi}{2} \quad \text { with } \lambda_{\phi}=0,1,2,3
\end{aligned}
$$

For these values, it holds that

$h=v=w=0$

Then (3) takes the form

$$
\begin{gathered}
-G m m_{0} z\left[\frac{1}{r_{1}^{3}}+\frac{1}{r_{2}^{3}}\right]-G m\left\{\sum _ { i = 1 } ^ { 2 } \left[\frac{-5 z\left(x-x_{i}\right)^{2} f}{r_{i}^{7}}\right.\right. \\
\left.\left.-\frac{5 z y^{2} g}{r_{i}^{7}}-\frac{5 z^{3} u}{r_{i}^{7}}+\frac{2 z}{r_{i}^{5}} u\right]\right\}=0
\end{gathered}
$$

or

$$
\begin{aligned}
& -G m m_{0} z\left[\frac{1}{r_{1}^{3}}+\frac{1}{r_{2}^{3}}\right]-G m\left\{\sum _ { i = 1 } ^ { 2 } \frac { z } { r _ { i } ^ { 7 } } \left[-5\left(x-x_{i}\right)^{2} f\right.\right. \\
& \left.\left.-5 y^{2} g-5 z^{2} u+2 r_{i}^{2} u\right]\right\}=0
\end{aligned}
$$

and finally

$$
\begin{gathered}
z\left\{-m_{0}\left[\frac{1}{r_{1}^{3}}+\frac{1}{r_{2}^{3}}\right]-\left\{\sum _ { i = 1 } ^ { 2 } \frac { 1 } { r _ { i } ^ { 7 } } \left[-5\left(x-x_{i}\right)^{2} f-5 y^{2} g\right.\right.\right. \\
\left.\left.\left.-5 z^{2} u+2 r_{i}^{2} u\right]\right\}\right\}=0
\end{gathered}
$$

This relation proves the existence of solutions on $x y$ plane $(z=0)$. Hereunder, we shall confine our study to the planar equilibrium positions. Then

$$
\begin{aligned}
& -G m\left\{\sum _ { i = 1 } ^ { 2 } \frac { 1 } { r _ { i } ^ { 5 } } \left[\left(x-x_{i}\right)^{2} \frac{\partial f}{\partial \psi}+y^{2} \frac{\partial g}{\partial \psi}\right.\right. \\
& \left.\left.+\left(x-x_{i}\right) y \frac{\partial h}{\partial \psi}\right]\right\}=0 \\
& -G m\left\{\sum _ { i = 1 } ^ { 2 } \frac { 1 } { r _ { i } ^ { 5 } } \left[\left(x-x_{i}\right)^{2} \frac{\partial f}{\partial \theta}+y^{2} \frac{\partial g}{\partial \theta}\right.\right. \\
& \left.\left.+\left(x-x_{i}\right) y \frac{\partial h}{\partial \theta}\right]\right\}+\left[\left(h_{a} \sin \phi+h_{b} \cos \phi\right) \cos \theta\right. \\
& \left.-h_{c} \sin \theta\right] \omega=0 \\
& -G m\left\{\sum _ { i = 1 } ^ { 2 } \frac { 1 } { r _ { i } ^ { 5 } } \left[\left(x-x_{i}\right)^{2} \frac{\partial f}{\partial \phi}+y^{2} \frac{\partial g}{\partial \phi}\right.\right. \\
& \left.\left.+\left(x-x_{i}\right) y \frac{\partial h}{\partial \phi}\right]\right\} \\
& +\left[\left(h_{a} \cos \phi-h_{b} \sin \phi\right) \sin \theta\right] \omega=0
\end{aligned}
$$


Table 1 Case $1\left(I_{1}=I_{2}\right)$

\begin{tabular}{|c|c|c|c|c|c|c|c|}
\hline \multicolumn{8}{|c|}{$I_{1}=I_{2}$} \\
\hline \multirow[t]{2}{*}{ Group } & \multicolumn{3}{|c|}{$h_{a}=0, h_{b} \neq 0, h_{c} \neq 0$} & \multirow[t]{2}{*}{ Group } & \multicolumn{3}{|c|}{$h_{a} \neq 0, h_{b}=0, h_{c} \neq 0$} \\
\hline & $\psi$ & $\theta$ & $\phi$ & & $\bar{\psi}$ & $\theta$ & $\phi$ \\
\hline \multirow[t]{4}{*}{ I } & 0 & 0 & \multirow{4}{*}{$\begin{array}{l}\frac{\pi}{2} \\
\frac{3 \pi}{2} \\
\frac{\pi}{2} \\
\frac{3 \pi}{2}\end{array}$} & \multirow[t]{4}{*}{$\mathrm{V}$} & 0 & 0 & 0 \\
\hline & 0 & 0 & & & 0 & 0 & $\pi$ \\
\hline & $\pi$ & 0 & & & $\pi$ & 0 & 0 \\
\hline & $\pi$ & 0 & & & $\pi$ & 0 & $\pi$ \\
\hline \multirow[t]{4}{*}{ II } & $\frac{\pi}{2}$ & 0 & $\frac{\pi}{2}$ & \multirow[t]{4}{*}{ VI } & $\frac{\pi}{2}$ & 0 & 0 \\
\hline & $\frac{\pi}{2}$ & 0 & $\frac{3 \pi}{2}$ & & $\frac{\pi}{2}$ & 0 & $\pi$ \\
\hline & $\frac{3 \pi}{2}$ & 0 & $\frac{\pi}{2}$ & & $\frac{3 \pi}{2}$ & 0 & 0 \\
\hline & $\frac{3 \pi}{2}$ & 0 & $\frac{3 \pi}{2}$ & & $\frac{3 \pi}{2}$ & 0 & $\pi$ \\
\hline \multirow[t]{4}{*}{ III } & 0 & $\pi$ & $\frac{\pi}{2}$ & \multirow[t]{4}{*}{ VII } & $\frac{\pi}{2}$ & $\pi$ & 0 \\
\hline & 0 & $\pi$ & $\frac{3 \pi}{2}$ & & $\frac{\pi}{2}$ & $\pi$ & $\pi$ \\
\hline & $\pi$ & $\pi$ & $\frac{\pi}{2}$ & & $\frac{3 \pi}{2}$ & $\pi$ & 0 \\
\hline & $\pi$ & $\pi$ & $\frac{3 \pi}{2}$ & & $\frac{3 \pi}{2}$ & $\pi$ & $\pi$ \\
\hline \multirow[t]{4}{*}{ IV } & $\frac{\pi}{2}$ & $\pi$ & $\frac{\pi}{2}$ & \multirow[t]{4}{*}{ VIII } & 0 & $\pi$ & 0 \\
\hline & $\frac{\pi}{2}$ & $\pi$ & $\frac{3 \pi}{2}$ & & 0 & $\pi$ & $\pi$ \\
\hline & $\frac{3 \pi}{2}$ & $\pi$ & $\frac{\pi}{2}$ & & $\pi$ & $\pi$ & 0 \\
\hline & $\frac{3 \pi}{2}$ & $\pi$ & $\frac{3 \pi}{2}$ & & $\pi$ & $\pi$ & $\pi$ \\
\hline
\end{tabular}

It is easily proved that when the Euler angles are integer multiples of $\pi / 2$, then

$\frac{\partial f}{\partial \psi}=\frac{\partial f}{\partial \theta}=\frac{\partial f}{\partial \phi}=\frac{\partial g}{\partial \psi}=\frac{\partial g}{\partial \theta}=\frac{\partial g}{\partial \phi}=\frac{\partial h}{\partial \theta}=0$

and in this case,

$-G m\left[\sum_{i=1}^{2} \frac{1}{r_{i}^{5}}\left(x-x_{i}\right) y\right] \frac{\partial h}{\partial \psi}=0$

$\left[\left(h_{a} \sin \phi+h_{b} \cos \phi\right) \cos \theta-h_{c} \sin \theta\right] \omega=0$

$-G m\left[\sum_{i=1}^{2} \frac{1}{r_{i}^{5}}\left(x-x_{i}\right) y\right] \frac{\partial h}{\partial \phi}$

$$
+\left[\left(h_{a} \cos \phi-h_{b} \sin \phi\right) \sin \theta\right] \omega=0
$$

The above (11) to (13) are verified for any triad $(\psi, \theta, \phi)$ of Euler angles that are integer multiples of $\pi / 2$ provided that the conditions written at the top of each table, are valid. We observe that each condition, $I_{1}=I_{2}, I_{1}=I_{3}$, or $I_{2}=I_{3}$ implies $f=g$. More specifically, we have the following cases:
Table 2 Case $2\left(I_{1}=I_{3}\right)$

\begin{tabular}{lcl}
\hline \multicolumn{3}{l}{$I_{1}=I_{3}$} \\
\hline$h_{a}=0, h_{b} \neq 0, h_{c}=0$ & \\
\hline$\psi$ & $\theta$ & $\phi$ \\
\hline 0 & $\frac{\pi}{2}$ & 0 \\
0 & $\frac{\pi}{2}$ & $\pi$ \\
$\frac{\pi}{2}$ & $\frac{\pi}{2}$ & 0 \\
$\frac{\pi}{2}$ & $\frac{\pi}{2}$ & $\pi$ \\
$\pi$ & $\frac{\pi}{2}$ & 0 \\
$\pi$ & $\frac{\pi}{2}$ & $\pi$ \\
$\frac{3 \pi}{2}$ & $\frac{\pi}{2}$ & 0 \\
$\frac{3 \pi}{2}$ & $\frac{\pi}{2}$ & $\pi$ \\
\hline
\end{tabular}

Case 1. $I_{1}=I_{2}$. Then $f=g=\frac{1}{2}\left(I_{2}-I_{3}\right)$. Equations (11) to (13) are verified for the combinations of the Euler angles which appear in Table 1.

Case 2. $I_{1}=I_{3}$. Then $f=g=\frac{1}{2}\left(I_{1}-I_{2}\right)$. Equations (11) to (13) are verified for the combinations of the Euler angles which appear in Table 2.

Case 3. $I_{2}=I_{3}$. Then $f=g=\frac{1}{2}\left(I_{3}-I_{1}\right)$ and (11)(13) are verified for the combinations of the Euler angles which appear in Table 3. 
Table 3 Case $3\left(I_{2}=I_{3}\right)$

\begin{tabular}{|c|c|c|}
\hline \multicolumn{3}{|c|}{$I_{2}=I_{3}$} \\
\hline \multicolumn{3}{|c|}{$h_{a} \neq 0, h_{b}=0, h_{c}=0$} \\
\hline$\psi$ & $\theta$ & $\phi$ \\
\hline 0 & $\frac{\pi}{2}$ & $\frac{\pi}{2}$ \\
\hline 0 & $\frac{\pi}{2}$ & 3 \\
\hline$\frac{\pi}{2}$ & $\frac{\pi}{2}$ & $\frac{\pi}{2}$ \\
\hline$\frac{\pi}{2}$ & $\frac{\pi}{2}$ & \\
\hline$\pi$ & $\frac{\pi}{2}$ & $\frac{\pi}{2}$ \\
\hline$\pi$ & $\frac{\pi}{2}$ & 3 \\
\hline$\frac{3 \pi}{2}$ & $\frac{\pi}{2}$ & $\frac{\pi}{2}$ \\
\hline$\frac{3 \pi}{2}$ & $\frac{\pi}{2}$ & \\
\hline
\end{tabular}

In view of the above values of $f$ and $g$, (1) and (2) take the form

$$
\begin{aligned}
& \omega^{2} x-G m\left[\sum_{i=1}^{2} \frac{\left(x-x_{i}\right)}{r_{i}^{3}}\right] \\
& -3 G \frac{m}{m_{0}} f\left[\sum_{i=1}^{2} \frac{\left(x-x_{i}\right)}{r_{i}^{5}}\right]=0 \\
& \omega^{2} y-G m y\left[\sum_{i=1}^{2} \frac{1}{r_{i}^{3}}\right]-3 G \frac{m}{m_{0}} y f\left[\sum_{i=1}^{2} \frac{y}{r_{i}^{5}}\right]=0
\end{aligned}
$$

We shall transform the above equations to dimensionless ones, in order to show their relation to those of a point-like mass. Thus, we first replace the principal central moments of inertia $I_{i}, i=1,2,3$ with their corresponding radii of gyration $\rho_{i}^{2}=\frac{I_{i}}{m_{0}}, i=1,2,3$. Then we have

$f=\frac{1}{2} m_{0}\left(\rho_{2}^{2}-\rho_{3}^{2}\right) \quad$ for case 1

$f=\frac{1}{2} m_{0}\left(\rho_{1}^{2}-\rho_{2}^{2}\right) \quad$ for case 2 and

$f=\frac{1}{2} m_{0}\left(\rho_{3}^{2}-\rho_{1}^{2}\right) \quad$ for case 3

Provided that

$a=\left|P_{1} P_{2}\right|, \quad \omega^{2}=\frac{G m}{a^{3}}$

and by introducing the transformations

$$
\begin{aligned}
& x=a x^{*}, \quad y=a y^{*}, \quad r_{i}=a r_{i}^{*} \\
& r=a r^{*} \quad \text { and } \quad f=a^{2} m_{0}\left(f^{*}\right)
\end{aligned}
$$

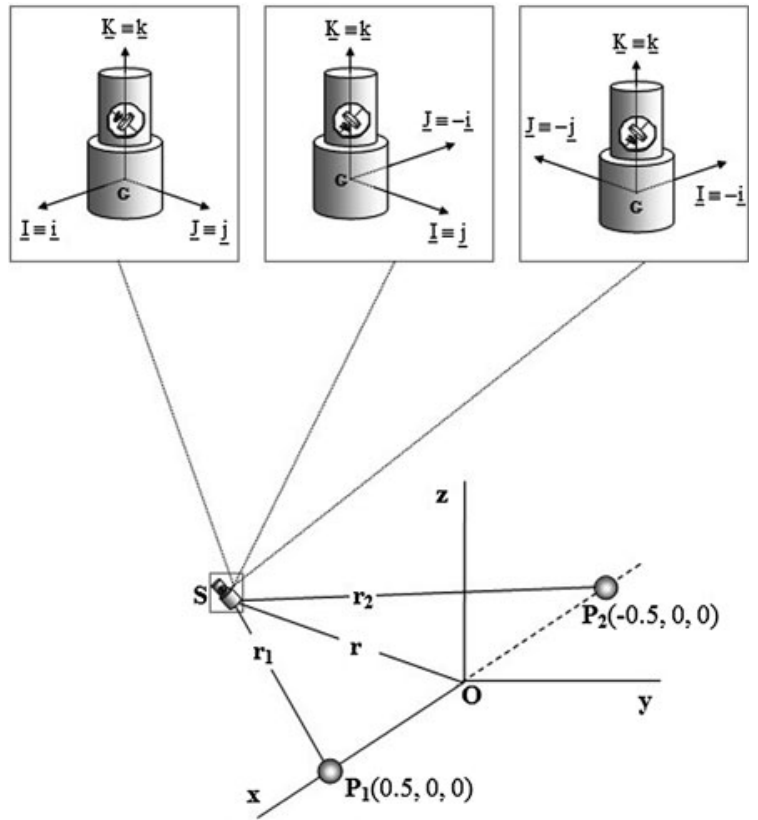

Fig. 2 The considered configuration with an inertially axisymmetric gyrostat $\left(I_{1}=I_{2}\right)$ when $h_{b}=0$. The framed pictures show three different orientations of attitude equilibrium obtained from Table 1

into (14) and (15), we obtain the dimensionless relations

$x-\left\{\left[\sum_{i=1}^{2} \frac{\left(x-x_{i}\right)}{r_{i}^{3}}\right]+\frac{3}{2} f\left[\sum_{i=1}^{2} \frac{\left(x-x_{i}\right)}{r_{i}^{5}}\right]\right\}=0$

$y-\left\{\left[\sum_{i=1}^{2} \frac{y}{r_{i}^{3}}\right]+\frac{3}{2} f\left[\sum_{i=1}^{2} \frac{y}{r_{i}^{5}}\right]\right\}=0$

from which we have omitted the asterisks. The orbital equilibria of $m_{0}$ with the constant orientations of the body-fixed axes are the solutions of the system of (16) and (17). It is evident that when $S$ has a spherical symmetry and the rotor is motionless in relation to the platform, then it will be

$I_{1}=I_{2}=I_{3}, \quad h_{a}=h_{b}=h_{c}=0$

$f=g=h=u=v=w=0$

and the problem reduces to the classic Copenhagen case where $S$ is a material point. Figure 2 shows some stationary solutions of an inertially axisymmetric gyrostat $S$ with $I_{1}=I_{2}$. The gyrostat rests on an orbital equilibrium position. The framed pictures show three 
different orientations of attitude equilibrium. The correspondence of the displayed orientations with Euler angles is obtained easily from Table 1.

Let us assume that $L_{e}\left(x_{e}, y_{e}, 0, \psi_{e}, \theta_{e}, \phi_{e}\right)$ is an equilibrium state of the gyrostat. If the conditions that correspond to this position are slightly disturbed, the variational equations of the planar translational motion will take the form:

$$
\begin{aligned}
& \delta \ddot{x}-2 \omega \delta \dot{y}=\sum_{q=x, y, \psi, \theta, \phi}\left(\frac{\partial E_{1}}{\partial q}\right)_{L_{e}} \delta q \\
& \delta \ddot{y}+2 \omega \delta \dot{x}=\sum_{q=x, y, \psi, \theta, \phi}\left(\frac{\partial E_{2}}{\partial q}\right)_{L_{e}} \delta q
\end{aligned}
$$

$E_{1}$ and $E_{2}$ are the right parts of (1) and (2) while their time derivatives are calculated on the position $L_{e}$.

Similarly, the equations of the rotational motion become

$$
\begin{aligned}
& {\left[\left(I_{1} \sin ^{2} \phi_{e}+I_{2} \cos ^{2} \phi_{e}\right) \sin ^{2} \theta_{e}+I_{3} \cos ^{2} \theta_{e}\right] \delta \ddot{\psi}} \\
& +\frac{1}{2}\left(I_{1}-I_{2}\right) \sin 2 \phi_{e} \sin \theta_{e} \delta \ddot{\theta}+I_{3} \cos \theta_{e} \delta \ddot{\phi} \\
& +\left[\left[\left(I_{1} \sin ^{2} \phi_{e}+I_{2} \cos ^{2} \phi_{e}\right)-I_{3}\right] \omega \sin 2 \theta_{e}\right. \\
& \left.+\left(h_{a} \sin \phi_{e}+h_{b} \cos \phi_{e}\right) \cos \theta_{e}-h_{c} \sin \theta_{e}\right] \delta \dot{\theta} \\
& +\left[\left(I_{1}-I_{2}\right) \omega \sin ^{2} \theta_{e} \sin 2 \phi_{e}+\left(h_{a} \cos \phi_{e}\right.\right. \\
& \left.\left.-h_{b} \sin \phi_{e}\right) \sin \theta_{e}\right] \delta \dot{\phi} \\
& =\sum_{q=x, y, \psi, \theta, \phi}\left(\frac{\partial E_{3}}{\partial q}\right)_{L_{e}} \delta q \\
& \frac{1}{2}\left(I_{1}-I_{2}\right) \sin 2 \phi_{e} \sin \theta_{e} \delta \ddot{\psi}+\left(I_{1} \cos ^{2} \varphi_{e}\right. \\
& \left.+I_{2} \sin ^{2} \varphi_{e}\right) \delta \ddot{\theta}-\left[\left[\left(I_{1} \sin ^{2} \phi_{e}+I_{2} \cos ^{2} \phi_{e}\right)\right.\right. \\
& \left.-I_{3}\right] \omega \sin 2 \theta_{e}+\left[\left(h_{a} \sin \phi_{e}+h_{b} \cos \phi_{e}\right) \cos \theta_{e}\right. \\
& \left.\left.-h_{c} \sin \theta_{e}\right]\right] \delta \dot{\psi}+\left[\left(I_{1}-I_{2}\right) \omega \cos 2 \phi_{e} \sin \theta_{e}\right. \\
& \left.+I_{3} \omega \sin \theta_{e}-\left(h_{a} \sin \phi_{e}+h_{b} \cos \phi_{e}\right)\right] \delta \dot{\phi} \\
& -\left[\left[\left(I_{1} \sin ^{2} \phi_{e}+I_{2} \cos ^{2} \phi_{e}\right)-I_{3}\right] \omega^{2} \cos 2 \theta_{e}\right. \\
& \left.-\left[\left(h_{a} \sin \phi_{e}+h_{b} \cos \phi_{e}\right) \sin \theta_{e}+h_{c} \cos \theta_{e}\right] \omega\right] \\
& \times \delta \theta-\left[\frac{1}{2}\left(I_{1}-I_{2}\right) \omega^{2} \sin 2 \theta_{e} \sin 2 \phi_{e}\right. \\
& \left.+\left(h_{a} \cos \phi_{e}-h_{b} \sin \phi_{e}\right) \omega \cos \theta_{e}\right] \delta \phi
\end{aligned}
$$

$$
\begin{aligned}
&-\frac{1}{2}\left[\left(I_{1} \sin ^{2} \phi_{e}+I_{2} \cos ^{2} \phi_{e}\right)-I_{3}\right] \omega^{2} \sin 2 \theta_{e} \\
&-\left[\left(h_{a} \sin \phi_{e}+h_{b} \cos \phi_{e}\right) \cos \theta_{e}-h_{c} \sin \theta_{e}\right] \omega \\
&= \sum_{q=x, y, \psi, \theta, \phi}\left(\frac{\partial E_{4}}{\partial q}\right)_{L_{e}} \delta q \\
& I_{3} \cos \theta_{e} \delta \ddot{\psi}+I_{3} \delta \ddot{\phi}-\left[\left(I_{1}-I_{2}\right) \omega \sin 2 \phi_{e} \sin ^{2} \theta_{e}\right. \\
&+ \\
&\left.+\left(h_{a} \cos \phi_{e}-h_{b} \sin \phi_{e}\right) \sin \theta_{e}\right] \delta \dot{\psi} \\
&-\left[\left(I_{1}-I_{2}\right) \omega \cos 2 \varphi_{e} \sin \theta_{e}+I_{3} \omega \sin \theta_{e}\right. \\
&\left.-\left(h_{a} \sin \phi_{e}+h_{b} \cos \phi_{e}\right)\right] \delta \dot{\theta} \\
&-\left[\frac{1}{2}\left(I_{1}-I_{2}\right) \omega^{2} \sin 2 \theta_{e} \sin 2 \phi_{e}\right. \\
&+\left.\left(h_{a} \cos \phi_{e}-h_{b} \sin \phi_{e}\right) \omega \cos \theta_{e}\right] \delta \theta \\
&-\left[\left(I_{1}-I_{2}\right) \omega^{2} \cos 2 \phi_{e} \sin ^{2} \theta_{e}-\left(h_{a} \sin \phi_{e}\right.\right. \\
&+\left.\left.h_{b} \cos \phi_{e}\right) \omega \sin \theta_{e}\right] \delta \phi \\
&- {\left[\frac{1}{2}\left(I_{1}-I_{2}\right) \omega^{2} \sin 2 \phi_{e} \sin ^{2} \theta_{e}+\left(h_{a} \cos \phi_{e}\right.\right.} \\
&=\left.\left.h_{b} \sin \phi_{e}\right) \omega \sin \theta_{e}\right] \\
& q= \sum_{, y, \psi}\left(\frac{\partial E_{5}}{\partial q}\right)_{L_{e}} \delta q \\
&
\end{aligned}
$$

In the last three equations, we denote with $E_{i}, i=$ $3,4,5$ the right parts of (4), (5), and (6), while their time derivatives are calculated on the equilibrium point $L_{e}$. By considering orientations $\psi_{e}, \theta_{e}, \phi_{e}$ that are integer multiples of $\pi / 2$, it is easily proven that

$$
\left(\frac{\partial E_{i}}{\partial q}\right)_{L_{e}}=0, \quad i=1,2, q=\psi, \theta, \phi
$$

and

$$
\left(\frac{\partial E_{j}}{\partial Q}\right)_{L_{e}}=0, \quad i=1,2, Q=x, y
$$

Then the variational equations can be written as

$$
\begin{aligned}
& \delta \ddot{x}-2 \omega \delta \dot{y}=\left(\frac{\partial E_{1}}{\partial x}\right)_{L_{e}} \delta x+\left(\frac{\partial E_{1}}{\partial y}\right)_{L_{e}} \delta y \\
& \delta \ddot{y}+2 \omega \delta \dot{x}=\left(\frac{\partial E_{2}}{\partial x}\right)_{L_{e}} \delta x+\left(\frac{\partial E_{2}}{\partial y}\right)_{L_{e}} \delta y
\end{aligned}
$$




$$
\begin{aligned}
& {\left[\left(I_{1} \sin ^{2} \phi_{e}+I_{2} \cos ^{2} \phi_{e}\right) \sin ^{2} \theta_{e}+I_{3} \cos ^{2} \theta_{e}\right] \delta \ddot{\psi}} \\
& +I_{3} \cos \theta_{e} \delta \ddot{\phi}+\left[\left(h_{a} \sin \phi_{e}+h_{b} \cos \phi_{e}\right) \cos \theta_{e}\right. \\
& \left.-h_{c} \sin \theta_{e}\right] \delta \dot{\theta}+\left(h_{a} \cos \phi_{e}-h_{b} \sin \phi_{e}\right) \sin \theta_{e} \delta \dot{\phi} \\
& =\sum_{q=\psi, \theta, \phi}\left(\frac{\partial E_{3}}{\partial q}\right)_{L_{e}} \delta q \\
& \left(I_{1} \cos ^{2} \phi_{e}+I_{2} \sin ^{2} \phi_{e}\right) \delta \ddot{\theta}+\left[\left(h_{a} \sin \phi_{e}\right.\right. \\
& \left.\left.+h_{b} \cos \phi_{e}\right) \cos \theta_{e}-h_{c} \sin \theta_{e}\right] \delta \dot{\psi} \\
& +\left[\left(I_{1}-I_{2}\right) \omega \cos 2 \phi_{e} \sin \theta_{e}+I_{3} \omega \sin \theta_{e}\right. \\
& \left.-\left(h_{a} \sin \phi_{e}+h_{b} \cos \phi_{e}\right)\right] \delta \dot{\phi} \\
& -\left\{\left[\left(I_{1} \sin ^{2} \phi_{e}+I_{2} \cos ^{2} \phi_{e}\right)-I_{3}\right] \omega^{2} \cos 2 \theta_{e}\right. \\
& -\left[\left(h_{a} \sin \phi_{e}+h_{b} \cos \phi_{e}\right) \sin \theta_{e}\right. \\
& \left.\left.+h_{c} \cos \theta_{e}\right] \omega\right\} \delta \theta-\left(h_{a} \cos \phi_{e}\right. \\
& \left.-h_{b} \sin \phi_{e}\right) \omega \cos \theta_{e} \delta \phi-\left[\left(h_{a} \sin \phi_{e}\right.\right. \\
& \left.\left.+h_{b} \cos \phi_{e}\right) \cos \theta_{e}-h_{c} \sin \theta_{e}\right] \omega \\
& =\sum_{q=\psi, \theta, \phi}\left(\frac{\partial E_{4}}{\partial q}\right)_{L_{e}} \delta q \\
& I_{3} \cos \theta_{e} \delta \ddot{\psi}+I_{3} \delta \ddot{\phi}-\left(h_{a} \cos \phi_{e}-h_{b} \sin \phi_{e}\right) \sin \theta_{e} \delta \dot{\psi} \\
& \text { - }\left[\left(I_{1}-I_{2}\right) \omega \cos 2 \phi_{e} \sin \theta_{e}+I_{3} \omega \sin \theta_{e}\right. \\
& \left.-\left(h_{a} \sin \phi_{e}+h_{b} \cos \phi_{e}\right)\right] \delta \dot{\theta} \\
& -\left(h_{a} \cos \phi_{e}-h_{b} \sin \phi_{e}\right) \omega \cos \theta_{e} \delta \theta \\
& -\left[\left(I_{1}-I_{2}\right) \omega^{2} \cos 2 \phi_{e} \sin ^{2} \theta_{e}\right. \\
& \left.-\left(h_{a} \sin \phi_{e}+h_{b} \cos \phi_{e}\right) \omega \sin \theta_{e}\right] \delta \phi \\
& -\left(h_{a} \cos \phi_{e}-h_{b} \sin \phi_{e}\right) \omega \sin \theta_{e} \\
& =\sum_{q=\psi, \theta, \phi}\left(\frac{\partial E_{5}}{\partial q}\right)_{L_{e}} \delta q
\end{aligned}
$$

We note that the variational equations that are related to the translation of the gyrostat, as well as those related to its rotation are uncoupled between them. Studying the stability of the gyrostat's rotational equilibria-for the various combinations of Euler angles accompanied by the corresponding conditions between $I_{1}, I_{2}, I_{3}$ and $h_{a}, h_{b}, h_{c}$ as classified on Tables 1,2 , and 3-it can be easily proven that for the cases considered it will always be

$\delta \psi(t)=\delta \dot{\psi}_{e} t+\delta \psi_{e}$
If, for example, we consider the triad $\psi=0, \theta=$ $\pi / 2, \phi=\pi / 2$ of Table 3, then (20) becomes

$I_{1} \delta \ddot{\psi}=0$

the solution of which has form (23). From all these, we come to the conclusion that the deviation of the disturbed solution is unbounded and consequently all the above equilibrium states of the gyrostat are unsteady.

\section{Conclusions}

The principal conclusion derived from the above analysis is that the stationary solutions of the small gyrostat which correspond to the integer multiple of $\pi / 2$ of the Eulerian angles are all unstable. Closing this article, we would like to emphasize anew the value of this model, especially as regards its ability to approach physical systems as described in detail in the introduction. In addition, stability appears as a necessary part of the equilibrium solutions. Especially in Gyro-dynamics the conclusions are quite important regardless of the actual character of equilibria (stable or unstable), because of their involvement to practical applications such as the transition of a satellite from an equilibrium position to another, the re-orientation of a vehicle, etc.

\section{References}

1. Cochran, J.E., Shu, P.H., Rews, S.D.: Attitude motion of asymmetric dual-spin spacecraft. J. Guid. Control Dyn. 5, 37-42 (1982)

2. Elipe, A., Lanchares, V.: Two equivalent problems: gyrostats in free motion and parametric Hamiltonians. Mech. Res. Commun. 24, 583-590 (1997)

3. Elipe, A., Arribas, M., Riaguas, A.: Complete analysis of bifurcations in the axial gyrostat problem. J. Phys. A Math. Gen. 30, 587-601 (1997)

4. El-Gohary, A.I.: On the stability of an equilibrium position and rotational motion of a gyrostat. Mech. Res. Commun. 24, 457-462 (1997)

5. El-Gohary, A.I.: On the stability of relative programmed motion of satellite-gyrostat. Mech. Res. Commun. 25, 371379 (1998)

6. Hall, C.D., Rand, R.H.: Spinup dynamics of axial dual-spin spacecraft. J. Guid. Control Dyn. 17, 30-37 (1994)

7. Hall, C.D.: Spinup dynamics of biaxial gyrostats. J. Astron. Sci. 43, 263-275 (1995)

8. Mavraganis, A.G., Kalvouridis, T.J.: The equilibrium attitudes of two gyrostats in syzygy with two attracting centers. In: Proceedings of the 2nd Astronomical Conference, Thessaloniki, June 29-July 1, 1995, pp. 609-614 (1996) 
9. Michalakis, D., Mavraganis, A.G.: The equilibrium configurations of the restricted problem of $2+2$ triaxial rigid bodies. Celest. Mech. Dyn. Astron. 63, 81-100 (1995)

10. Pascal, M.: Problème restreint des trois corps appliqué à un gyrostat. Celest. Mech. Dyn. Astron. 11(3), 319-336 (1975)

11. Roy, A.: Orbital Motion. Hilger, Bristol (1979)

12. Rubanovskii, V.N.: On the relative equilibrium of a gyrostat satellite in the generalized limited circular problem of three bodies. Prikl. Mat. Meh. 45, 360-367 (1982)

13. Tong, X., Tabarrok, B., Rimrott, F.P.J.: Chaotic motion of an asymmetric gyrostat in the gravitational field. Int. J. Non-Linear Mech. 30, 191-203 (1995)

14. Tsogas, V., Mavraganis, A.G.: Some equilibrium of the restricted three-body problem with axial symmetric primaries and a gyrostat as infinitesimal mass. Celest. Mech. Dyn. Astron. 85(4), 293-309 (2003)

15. Vera, J.A., Vigueras, A.: Libration points of a gyrostat in a generalized restricted three-body problem: linear stability. Adv. Astronaut. Sci. 123(III), 2775-2794 (2006)

16. Vera, J.A., Vigueras, A.: Hamiltonian dynamics of a gyrostat in the n-body problem: relative equilibria. Celest. Mech. Dyn. Astron. 94(30), 289-315 (2006)

17. Vera, J.A.: Eulerian equilibria of a triaxial gyrostat in the three-body problem: rotational Poisson dynamics in Eulerian equilibria. Nonlinear Dyn. 55(1-2), 191-201 (2009) 